\title{
A novel compound heterozygous mutation in VARS2 in a newborn with mitochondrial cardiomyopathy: a case report of a Chinese family
}

Keze Ma ${ }^{1,2+}$, Mingyu Xie ${ }^{1,2+}$, Xiaoguang He ${ }^{1,2}$, Guojun Liu' ${ }^{1,2}$, Xiaomei Lu², Qi Peng ${ }^{2}$, Baimao Zhong ${ }^{1,2^{*}}$ and Ning $\mathrm{Li}^{1,2^{*}}$

\begin{abstract}
Background: Genetic defects in the mitochondrial aminoacyl-tRNA synthetase are important causes of mitochondrial disorders. VARS2 is one of the genes encoding aminoacyl-tRNA synthetases. Recently, an increasing number of pathogenic variants of VARS2 have been reported.

Case presentation: We report the novel compound heterozygous pathogenic VARS2 mutations c.643 C > T (p. His215Tyr) and c.1354 A > G (p. Met452Val) in a female infant who presented with poor sucking at birth, poor activity, hyporeflexia, hypertonia, persistent pulmonary hypertension of newborn (PPHN), metabolic acidosis, severe lactic acidosis, expansion and hypertrophic cardiomyopathy. These heterozygous mutations were carried individually by the proband's parents and elder sister; the two mutations segregated in the family and were the cause of the disease in the proband.The c.643 C > T ( $p$. His215Tyr) mutation was not described in the ExaC, GNomAD and 1000 Genomes Project databases, and the frequency of c.1354 A > G (p. Met452Val) was < 0.001 in these gene databases. The two mutated amino acids were located in a highly conserved region of the VARS2 protein that is important for its interaction with the cognate tRNA. The two missense mutations were predicted by online tools to be damaging and deleterious.
\end{abstract}

Conclusions: Our report expands the spectrum of known pathogenicVARS2 variants associated with mitochondrial disorders in humans.VARS2 deficiency may cause a severe neonatal presentation with structural cardiac abnormalities.

Keywords: Mitochondrial disorders, Cardiomyopathy, VARS2, Neonate

\section{Background}

Mitochondrial diseases (MDs) are multisystem disorders that are caused by inherited or acquired mutations in either the mitochondrial DNA or nuclear DNA known to encode mitochondrial proteins [1]. Mitochondrial aminoacyl-tRNA synthetases (mt-aaRSs) are key enzymes in mitochondrial protein synthesis that catalyze the binding of amino acids to their specific tRNAs (http://www.Uniprot.org).

The VARS2 gene is one of the genes encoding mt-aaRSs. Few individual patients with VARS2 deficiencies have been described with specific phenotypes. The

\footnotetext{
* Correspondence: zbomao@163.com; mean163@163.com

${ }^{\dagger}$ Keze Ma and Mingyu Xie contributed equally to this work.

${ }^{1}$ Department of Neonatal Intensive Care Unit, Dongguan Children's Hospital,

Dongguan 523325, Guangdong, China

Full list of author information is available at the end of the article
}

phenotypes present at any time during life and demonstrate great clinical heterogeneity. Mutations in the VARS2 gene are associated with encephalomyopathy or cardiomyopathy and result in chronic disability and a poor prognosis [2-4]. These patients can present with structural brain abnormalities, hypotonia, psychomotor delay, seizures, feeding difficulty, severe lactic acidosis, hypertrophic cardiomyopathy, and abnormal cranial magnetic resonance imaging (MRI) $[2,5]$.

In the present study, we found a novel compound heterozygous variant of the VARS2 gene [c.643 $\mathrm{C}>\mathrm{T}$ (p.His215Tyr) plus c.1354 A>G (p.Met452Val)] in the proband by targeted capture and sequencing. p.His215 and p.Met452 were located in the aminoacyl-tRNA synthetase (also known as amino acid translation or aminoacyl-tRNA 
ligase). The proband was diagnosed with mitochondrial cardiomyopathy and presented with poor sucking, hypertonia, severe lactic acidosis, hypertrophic cardiomyopathy and severe pulmonary hypertension and died on the sixteenth day of life due to cardiac arrest.We present detailed clinical data and further delineate the phenotype associated with this disease.

\section{Case presentation}

The female proband was the fifth child of nonconsanguineous parents of Han Chinese descentand was born at 38 weeks gestation by Cesarean section delivery due to a uterine scar to a 30-year-old woman following an uneventful pregnancy. The first child of the parents was an unexplained spontaneous abortion, and the second child was an abortion due to a heterotopic pregnancy. The third child died soon after birth with an unknown diagnosis in a grass-roots hospital. The fourth child had a normal phenotype (Fig. 1). The family had no metabolic disorders. The proband had no postnatal adaptation, and the Apgar score was 10 at $1 \mathrm{~min}$. Her birth weight was $2.64 \mathrm{~kg}$ (between the $3^{\text {rd }}$ and $10^{\text {th }}$ percentiles). Her head and abdominal circumferences were $32 \mathrm{~cm}\left(10^{\text {th }}\right.$ percentile). Her length was $49 \mathrm{~cm}$ ( $50^{\text {th }}$ percentile).

The newborn presented with poor sucking at birth and was transferred to the neonatal intensive care unit due to poor vigor, groaning, shortness of breath and cyanosis, and shock at the sixth day of life. Laboratory analyses found metabolic acidosis and severe lactic acidosis based on the arterial blood gas results, including $\mathrm{pH}$ 7.167, $\mathrm{pCO}_{2} 16.9 \mathrm{mmHg}, \mathrm{pO}_{2} 50.4 \mathrm{mmHg}, \mathrm{HCO}_{3}-$ $6.2 \mathrm{mmol} / \mathrm{L}, \mathrm{BE}-22.6 \mathrm{mmol} / \mathrm{L}$, and lactate $13.7 \mathrm{mmol} /$ $\mathrm{L}$ (reference ranges: arterial $\mathrm{pH}$ 7.35-7.45, $\mathrm{pCO}_{2} 35-$ $45 \mathrm{mmHg}, \mathrm{pO}_{2} 60-90 \mathrm{mmHg}, \mathrm{HCO}_{3}-21-24 \mathrm{mmol} / \mathrm{L}$, $\mathrm{BE}-3-3 \mathrm{mmol} / \mathrm{L}$, and lactate $\leq 2.5 \mathrm{mmol} / \mathrm{L}$ ). Albumin, normal saline and vasoactive agents (dopamine and dobutamine) were used to improve circulation. The acidosis was treated with sodium bicarbonate, but the

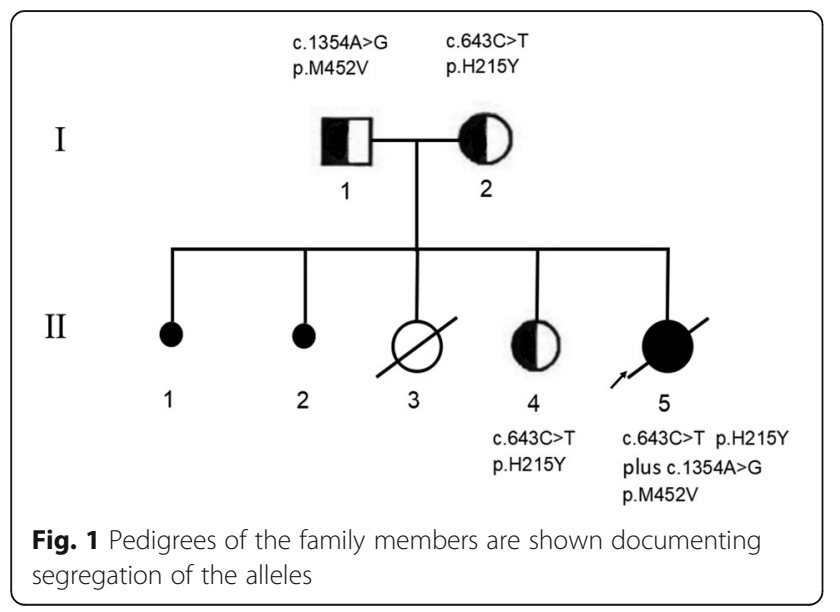

plasma lactate acid was still $15.6 \mathrm{mmol} / \mathrm{L}$. Coenzyme A and adenosine triphosphate were used to improve the acidosis, but the plasma lactate acid fluctuated between 3.0 and $10.9 \mathrm{mmol} / \mathrm{L}$. She did not present pronounced urinary lactate. Further metabolic work-up revealed an abnormal increase in $\mathrm{N}$-acetyl tyrosine-2 in the urinary organic acid test, but no abnormal acylcarnitine profiles and amino acids were detected.

Echocardiography revealed the presence of right atrial and ventricular expansion, right ventricular hypertrophy, a normal ventricular ejection function, interventricular septum thickening, tricuspid regurgitation and severe pulmonary hypertension at admission. The percutaneous blood saturation revealed 15\% variation before and after the catheter. The PPHN was treated with sidenafil. The electrocardiograph showed nodal tachycardia, right ventricular hypertrophy and movement of the ST segment down $0.1 \mathrm{mv}$ at V1 and V2. The Non-Invasive Cardiac System showed tachycardia, a high cardiac output, a reduction in left ventricle systolic function, and high total peripheral resistance at admission. The chest CT scan and three-dimensional reconstruction displayed coarctation of the aorta and right lung pneumonia. The renal and hepatic function tests, creatine kinase, lactate dehydrogenase, ammonia and total homocysteine were normal. The abdominal ultrasound was also normal.

On the physical examination, there were some signs of shock and mild dehydration. The neurological examination revealed poor reactivity, bregma depression with normal size, hyporeflexia and mild hypermyotonia. No seizures, nystagmus, laryngeal stridor or apnea were present in the neurological signs. The amplitude-integrated electroencephalogram (aEEG) indicted a mild abnormality (she showed no obvious sleep-wake cycles). The brain ultrasonic examination revealed mild echo enhancement on the side of the bilateral paraventricular parenchyma, a left-ependymal cyst and a right-choroid plexus cyst. The laboratory investigations showed that she had mild anemia. The child died on the sixteenth day of life due to cardiac arrest.

\section{Materials and methods}

\section{DNA extraction and sequencing}

Genomic DNA from the family was extracted from peripheral whole blood samples using the SolPure Blood DNA kit (Magen) according to the manufacturer's instructions. The genomic DNA of the three patients was fragmented with the Q800R Sonicator (Qsonica) to generate 300-500-bp fragments.

The paired-end libraries were prepared according to the Illumina library preparation protocol. Custom-designed NimbleGen SeqCap probes (Roche NimbleGen, Madison, WI, USA) were used for in-solution hybridization to enrich the target sequences. The enriched DNA samples 
were indexed and sequenced on a NextSeq500 sequencer (Illumina, San Diego, CA, USA) with 100,150 cycles of single end reads according to the manufacturer's protocols.

DNA from the patient's sister was validated by Sanger sequencing according to parents' and the proband's sequencing results.

\section{Variant annotation and interpretation}

The primary data were transformed to the FASTA format after the image analysis, and base calling was conducted using the Illumina Pipeline. The data were filtered to generate 'clean reads' by removing adapters and low-quality reads (Q20). The sequencing reads were mapped to the reference human genome version hg19 (2009-02 release, http://genome.ucsc.edu/). Nucleotide changes observed in the aligned reads were called and reviewed using the NextGENe software (SoftGenetics, State College, PA, USA). In addition to the detection of deleterious mutations and novel single nucleotide variants, a coverage-based algorithm developed in-house (eCNVscan) was used to detect large exonic deletions and duplications. The normalized coverage depth of each exon of a test sample was compared with the mean coverage of the same exon in the reference file to detect copy number variants (CNVs).

Sequence variants were annotated using population and literature databases, including 1000 Genomes (http:// www.1000genomes.org/), dbSNP (http://www.ncbi.nlm.nih.gov/), GnomAD (http://gnomad.broadinstitute.org/), Clinvar (https://www.st-va.ncbi.nlm.nih.gov/clinvar/), HG MD (http://www.hgmd.cf.ac.uk/ac/index.php) and OMIM (https://www.omim.org/). Online software (https://www.u niprot.org/) was used to analyze the protein structure, predict the conserved and functional domains and perform a multiple sequence alignment. Variant interpretation was performed according to the American College of Medical Genetics (ACMG) guidelines [6]. The possible pathogenicity was predicted according to the online tools MutationTaster, PolyPhen-2, SIFT and MutationAssessor.

\section{Homology modeling}

Homology modeling of the VARS2 gene in amino acid region 129-1079 was performed by employing the Protein Data Bank (PDB) structure 1gax.1B from UniProt and SwissModel as the template. All side chain atoms in the template structure were deleted. Novel sites and different functional regions were labeled using Chimera 1.12. We show the location of the compound heterozygous mutation on the partial VARS2 protein structure (Fig. 3b).

\section{Results}

\section{Sequence analysis}

The DNA sequence analysis showed that the proband presented a compound heterozygous missense mutation c.643 C > T (p.His215Tyr) in exon 5 and a missense mutation c.1354 A $>$ G (p.Met452Val) in exon 13 of the VARS2 gene (NM_001167734.1). The c.643 C>T (p. His215Tyr) mutation was not described in the ExaC, GNomAD and 1000 Genomes Project databases, and the frequency of c.1354 A > G (p. Met452Val) was $<0.001$ in these gene databases. Her mother and sister were heterozygous carriers of the c.643 $\mathrm{C}>\mathrm{T}$ (p.His215Tyr) mutation, and the father was a heterozygous carrier of the c.1354 A > G (p.Met452Val) mutation (Fig. 2).

The two missense mutations c.643 C > T (p.His215Tyr) and c.1354 A > G (p.Met452Val) are located in regions important for the interaction of the VARS2 protein with the cognate tRNA. We also analyzed this amino acid region in different species. The result indicated that c.643 C > T (p.His215Tyr) and c.1354 A > G (p.Met452Val) were located in a highly conserved region of the protein (Fig. 3a). The variant VARS2: c.643 C $>\mathrm{T}$ (p.His215Tyr) was predicted to be "disease causing" by MutationTaster, "probably damaging" by PolyPhen-2 with a score of 1.000 (sensitivity: 0.00; specificity: 1.00), "Affect protein function" by SIFT, and "high" on "function impact" by MutationAssessor. The variant VARS2: c.1354 A > G (p.Met452Val) was predicted to be "disease causing" by MutationTaster, "medium" on "function impact" by MutationAssessor, "tolerated" by SIFT, and "benign" by Polyphen-2 with a score of 0.104 (sensitivity: 0.91; specificity: 0.69).However, two amino acid mutations in a highly conserved region of VARS2 may seriously impact normal protein function in mammals.

\section{Discussion and conclusions}

Variants of the VARS2 gene, which is located on chromosome 6p21 and encodes a mt-aaRS, are one cause of MDs, although this same gene deficiency may be associated with different clinical phenotypes (Table 1). Recent reports identified variants in this gene associated with mitochondrial encephalopathy, encephalocardiomyopathy and cardiomyopathy [2]. However, how the same gene deficiency can cause these heterogeneous phenotypes remains poorly understood. One hypothesis indicated the gene was associated with the tissue-related amino acid concentrations. Our report further expands the clinical phenotype related to VARS2 deficiency. Here, we described a newborn with novel compound heterozygous VARS2 mutations[c.643 C > T (p.His215 Tyr) and c.1354 A > G (p.Met452Val)]who initially presented with poor sucking, hypertonia, respiratory distress, cyanosis, persistent pulmonary hypertension of newborn, right atrial and ventricular hypertrophy, interventricular septum thickening, tricuspid regurgitation, metabolic acidosis and severe lactic acidemia and was diagnosed with neonatal cardiomyopathy. 


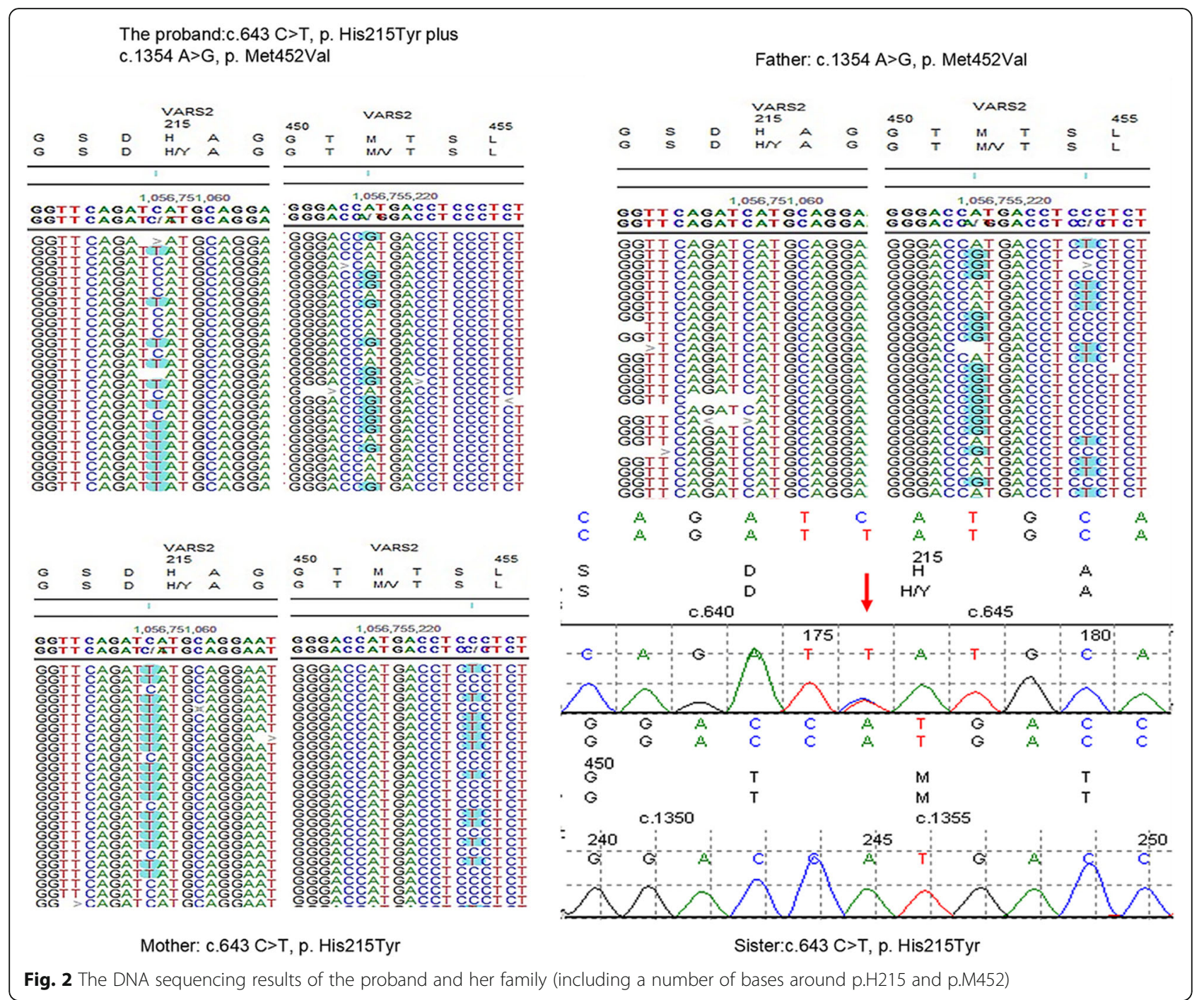

Most recent studies have indicated patients primarily present with a phenotype characterized by severe neonatal encephalocardiomyopathy with hypotonia, poor sucking, respiratory failure, lactic acidosis and abnormal heart anatomy $[2,5,7]$. For example, Fabian B. et al. [5] reported that a newborn with compound heterozygous variants c.601 C>T (p.Arg201Trp) plus c.1100 C > T (p.Thr367lle) presented with hypertonia, focal seizures, and apnea and developed lactic acidosis. Cardiological ultrasound revealed severe hypertrophic left ventricular dilations and reduced function. The echocardiographic (ECG) examination revealed a severe hypertrophic left ventricle accompanied by moderate dilation and a decrease of the ejection fraction. The brain MRI displayed structural brain abnormalities at the age of five weeks, such as hypoplasia of the corpus callosum and the cerebellum and edema of the brain stem and the frontal white matter. Francesco B et al. [2] described a newborn
(c.2557-2A > G plus c.1100 C > T, p.Thr367Ile) who presented with hypotonia, stridor, apnea, hyporeflexia, irritability and intermittent lactic acidosis. The cardiological ultrasound revealed biventricular hypertrophy and pericardial diffusion. The head MRI displayed cerebellar atrophy and a lactate peak at the MR. The described patient herein initially presented with poor sucking, hypertonia, PPHN, severe lactic acidosis and abnormal heart anatomy. However, the patient lacked a head MRI due to the parents' refusal.

The most frequent variant was homozygous variant c.1100C > T (p.Thr367lle) according to previous reports, including six cases to date [2,7]. However, their clinical signs were not exactly consistent. Most patients with homozygous variant c.1100C $>\mathrm{T}$ (p.Thr367lle) presented with hypertonia, developmental delays, microcephaly, and seizures but no cardiomyopathy $[2,7]$. These data could suggest that the homozygous variant c.1100C $>\mathrm{T}$ 
a

Human

Equus

Sus scrofa

Ailuropoda

Canis

Heterocephalus

Rerio

Takifugu

Harpegnathos
H215Y

$\downarrow$
M452V

1

WV P G S D HA G A T Q A. . . I A E D G TM

WV P G S DHA G A T Q V. . I A E D G TM

WV P G S D HA G I A Q A. . . I A E D G TM

WV P G S DHA G A T QA. . . I A E D G T M

WI P G S D HA G I A Q A. . . I A E D G TM

WV P G S DHA G A T QA. . I E E D G TM

WV P G C DHA G I A Q S . . I G G N G TM

WV P G C D HA G A T QT. . I G G D T M

WI P G L DHA G A T Q V. . I N E D G NM

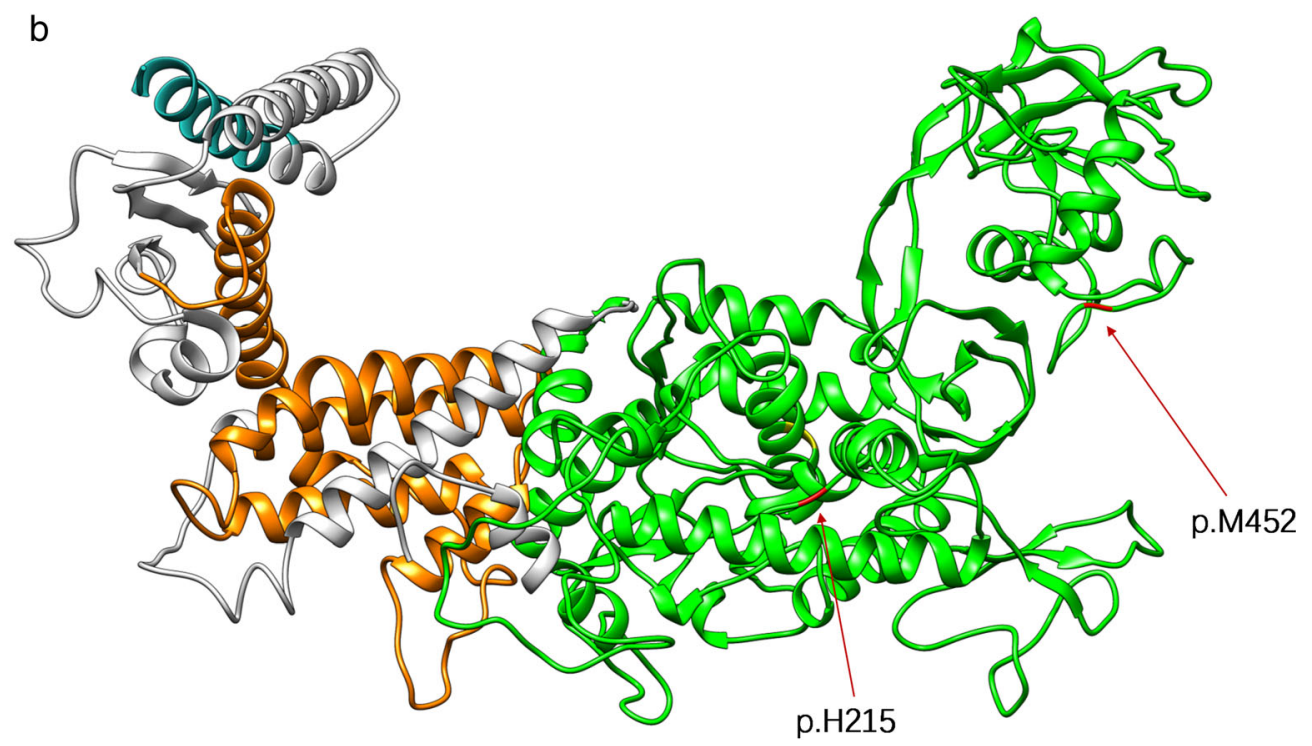

C

Valine-tRNA ligase, colied

ATP binding site(661)

Valine-tRNA ligase, coiled
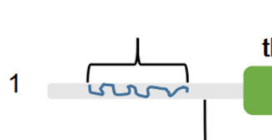

tRNA-synthetase domain(143-759)

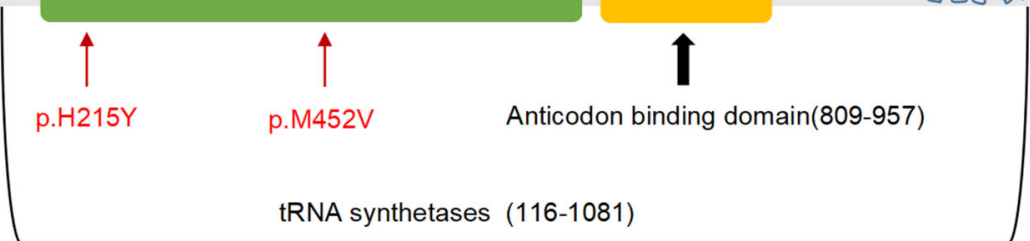

1093

Fig. 3 Schematic diagram and molecular model of the VARS2 gene. a VARS2 sequence alignment among representative species around the sites of the missense mutations (p.H215Y and p.M452 V). $\mathbf{b}$ Homology model of VARS2 (partial amino acids from positions 129 to 1079). Different colors represent different functional regions: the compound heterozygous mutation (p.His215 and p.Met452) is shown in red, the valine-tRNA ligase region in blue, the ATP biding site in yellow, the tRNA-synthetase domain in green and the anticodon binding domain in orange. $\mathbf{c}$ Schematic representation of the VARS2 protein. The novel compound heterozygous mutation reported here coincides with the tRNA-synthetase domain 


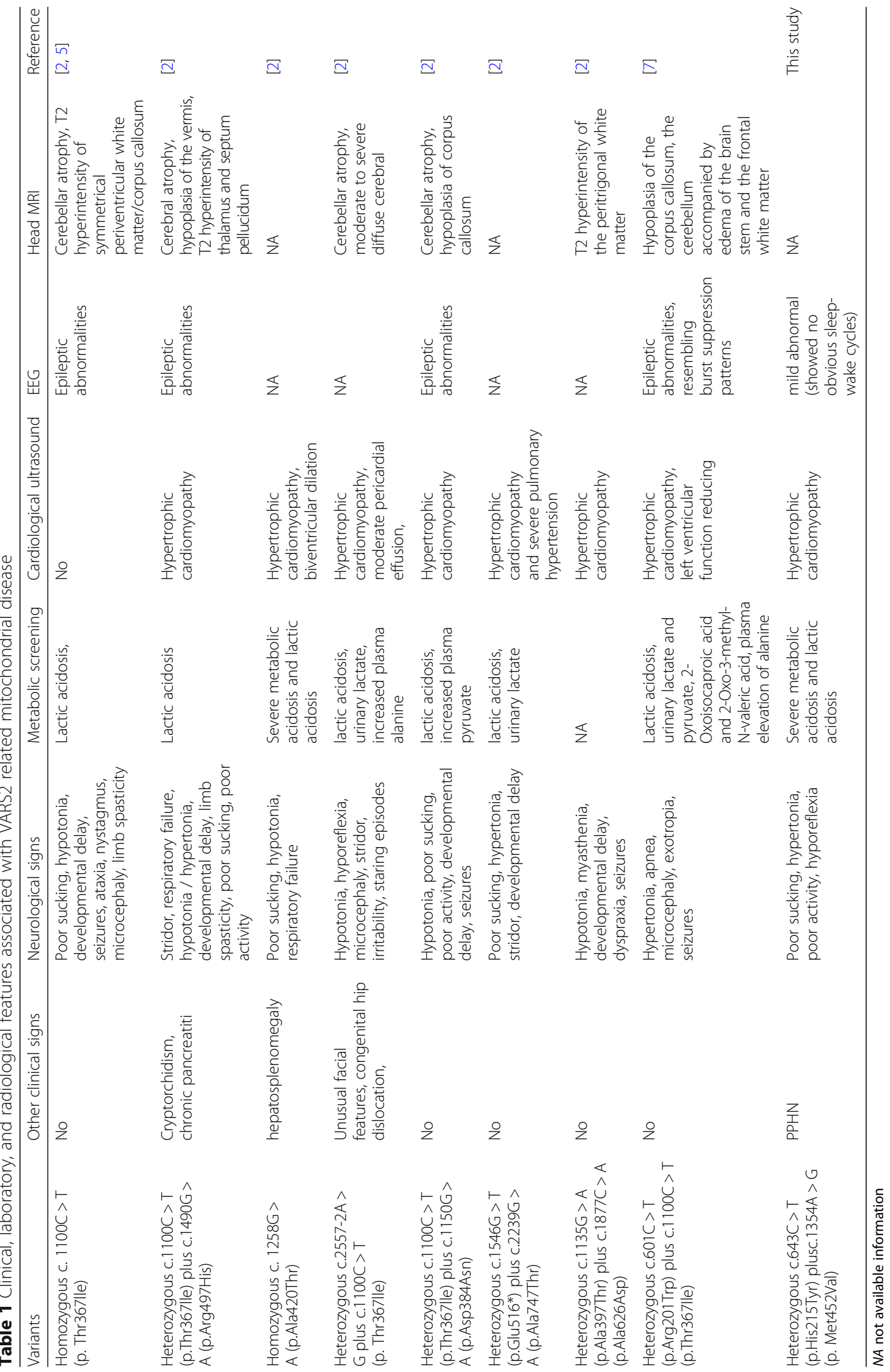


(p.Thr367lle) might have a lesser effect on the heart. Hypertonia, seizures, developmental delays, and lactic acidosis seem to be common signs of VARS2-related $\mathrm{MD}$, but seizures and developmental delays usually appear later in life $[2,5,7]$. Our case died at six days of life and did not present epilepsy or developmental delays, which might be due to the improper timing. Additionally, our proband initially presented with hypertonia but not hypotonia, which was similar to previous patient with compound heterozygous variantsc.601 C > T (p.Arg201Trp) plus c.1100C $>\mathrm{T}$ (p.Thr367lle) [5] or c.1546 G > T (p.Glu516) plus c.2239G > A (p.Ala367Thr) [2]. PPHN was the notable feature of our proband, which was less often described in previous reports. Only a patient with the compound heterozygous variants c.1546 G > T (p.Glu516) plus c.2239G > A (p.Ala367Thr) was described with severe pulmonary hypertension and death at 19 months [2].

Most patients with variants of VARS2 have a poor prognosis. However, few patients die during the neonatal period. Francesco et al. [2] reported a case of a premature death with a homozygous mutation c.1258 G > T (p.Ala420Thr) in VARS2 who presented with hypotonia and poor sucking at birth, followed by respiratory failure, severe metabolic acidosis, lactic acidosis and hypertrophic cardiomyopathy. He died on the ninth day of life. The present report describes a newborn death at sixteen days of life.

p.His215 and p.Met452 are located in the tRNAsynthetase domain (Fig. 3c). Previous studies have indicated that most patients with VARS2 variants in that region have great clinical impairment or a poor prognosis $[2,5,7]$. Additionally, p.His 215 and p.Met452 involve residues that are highly conserved among phylogenetically distant organisms, allowing us to suggest that these variants may affect the 3-dimensional conformation of the VARS2 protein. However, further study is needed to confirm this hypothesis. This finding highlights the functional importance of the site and suggests that variants may change the structure of the VARS2 protein and cause defective binding of the tRNA. Based on the ACMG criteria [6], there is some evidence for pathogenicity of the novel compound heterozygous mutations, including two moderate (PM1 and PM2) and four supporting (PP1 to PP4) pieces of evidence. Therefore, the two novel mutations in VARS2 may be classified as likely pathogenic variants. In future studies, we will focus on developing transgenic animal models.

The discovery of novel variants further expands the spectrum of known VARS2 mutations in humans.VARS2 deficiency may cause a severe neonatal presentation with structural cardiac abnormalities. In future studies, we will focus on developing transgenic animal models carrying the variants to characterize the mechanism by which VARS2 deficiency leads to mitochondrial disease.

\section{Abbreviations}

ACMG: American College of Medical Genetics; ATP: Adenosine triphosphate; ECG: Echocardiography; MDs: Mitochondrial disorders; MRC: Mitochondrial respiratory chain; MRI: Magnetic resonance imaging; mt-aaRS: Mitochondrial aminoacyl-tRNA synthetases; mtDNA: Mitochondrial DNA; nDNA: Nuclear DNA; OXPHOS: Oxidative phosphorylation; PDB: Protein data bank;

PPHN: Persistent pulmonary hypertension of newborn

\section{Acknowledgements}

The authors thank all family members for their cooperation during this work.

The authors declare that they have no competing interests

\section{Funding}

The study was supported by the Dongguan Bureau of Science andTechnology for the City Key Programs of Science and Technology(Project Numbers: 2014108101027 and 2015108101031).

\section{Availability of data and materials}

All relevant data are included in the manuscript. The datasets used and/or analyzed during the current study are available from the corresponding author upon request.

\section{Authors' contributions}

KZM, MYX, BMZ, and NL conceived and designed the work; KZM, MYX, XGH, $G J, X M L, Q P, B M Z$, and NL performed the experiments, KZM, MYX, XGH, GJL, $B M Z$, and NL analyzed the data. KZM, MYX, BMZ, and NL wrote the manuscript. All authors read and approved the final manuscript.

\section{Ethics approval and consent to participate}

The study was approved by the Ethics Committee of Dongguan Children's Hospital in agreement with the Declaration of Helsinki. Informed consent was obtained from the parents of the study subject.

Consent for publication

All members of the family signed a written consent form for publication of all data.

Competing interests

\section{Publisher's Note}

Springer Nature remains neutral with regard to jurisdictional claims in published maps and institutional affiliations.

\section{Author details}

${ }^{1}$ Department of Neonatal Intensive Care Unit, Dongguan Children's Hospital, Dongguan 523325, Guangdong, China. ${ }^{2}$ Department of Medical and Molecular Genetics, Dongguan Institute of Pediatrics, Dongguan 523325, Guangdong, China.

Received: 11 June 2018 Accepted: 20 September 2018

Published online: 20 November 2018

References

1. Spinazzola A, Zeviani M. Mitochondrial diseases: a cross-talk between mitochondrial and nuclear genomes. Adv Exp Med Biol. 2009;652:69-84.

2. Bruni F, Di Meo I, Bellacchio E, Webb BD, McFarland R, et al. Clinical, biochemical, and genetic features associated with VARS2-related mitochondrial disease. Hum Mutat. 2018;39:563-78.

3. Chae YS, Lee SJ, Moon JH, et al. VARS2 V552V variant as prognostic marker in patients with early breast cancer. Med Oncol. 2011;28:1273-80.

4. Alsemari A, Al-Younes B, Goljan E, Kang BW, Kim JG, et al. Recessive VARS2 mutation underlies a novel syndrome with epilepsy, mental retardation, short stature, growth hormone deficiency, and hypogonadism. Hum Genomics. 2017:11:28.

5. Baertling F, Alhaddad B, Seibt A, Budaeus S, Meitinger T, et al. Neonatal encephalocardiomyopathy caused by mutations in VARS2. Metab Brain Dis. 2017;32:267-70 
6. Richards S, Aziz N, Bale S, Bick D, Das S, et al. Standards and guidelines for the interpretation of sequence variants: a joint consensus recommendation of the American College of Medical Genetics and Genomics and the Association for Molecular Pathology. Genet Med. 2015;17:405-24.

7. Pereira S, Adrião M, Sampaio M, Basto MA, Rodrigues E, et al. Mitochondrial encephalopathy: first Portuguese report of a VARS2 causative variant.JIMD Rep2018, [Epub ahead of print].

Ready to submit your research? Choose BMC and benefit from:

- fast, convenient online submission

- thorough peer review by experienced researchers in your field

- rapid publication on acceptance

- support for research data, including large and complex data types

- gold Open Access which fosters wider collaboration and increased citations

- maximum visibility for your research: over $100 \mathrm{M}$ website views per year

At $B M C$, research is always in progress.

Learn more biomedcentral.com/submissions 